\title{
Capsule endoscopy with flexible spectral imaging color enhancement reduces the bile pigment effect and improves the detectability of small bowel lesions
}

Eiji Sakai ${ }^{1}$, Hiroki Endo ${ }^{1 *}$, Shingo Kato ${ }^{1}$, Tetsuya Matsuura², Wataru Tomeno², Leo Taniguchi ${ }^{2}$, Takashi Uchiyama², Yasuo Hata ${ }^{2}$, Eiji Yamada', Hidenori Ohkubo ${ }^{1}$, Takuma Higrashi ${ }^{1}$, Kunihiro Hosono ${ }^{1}$, Hirokazu Takahashi ${ }^{1}$ and Atsushi Nakajima'

\begin{abstract}
Background: Capsule endoscopy with flexible spectral imaging color enhancement (CE-FICE) has been reported to improve the visualization and detection of small-bowel lesions, however, its clinical usefulness is still not established. Therefore, we conducted a study to evaluate whether CE-FICE contributes to improve the detectability of small-bowel lesions by CE trainees.

Methods: Four gastroenterology trainees without prior CE experience were asked to read and interpret 12 CE videos. Each of the videos was read by conventional visualization method and under three different FICE settings. To evaluate whether the lesion recognition ability of the CE trainees could be improved by the FICE technology, the lesion detection rate under each of the three FICE settings was compared with that by conventional CE. CE trainees tend to miss small-bowel lesions in bile-pigment-positive condition, therefore we evaluated whether CE-FICE contributes to reducing the bile-pigment effect. The bile-pigment condition was determined by the color values around the small-bowel lesions according to the results of the receiver-operating-characteristic analysis. Moreover, we also evaluated whether poor bowel preparion might affect the accuracy of lesion recognition by CE-FICE.

Results: Of a total of 60 angioectasias, CE trainees identified 26 by conventional CE, 40 under FICE setting 1, 38 under FICE setting 2, and 31 under FICE setting 3. Of a total of 82 erosions/ulcerations, CE trainees identified 38 by conventional CE, 62 under FICE setting 1,60 under FICE setting 2, and 20 under FICE setting 3. Compared with conventional CE, FICE settings 1 and 2 significantly improved the detectability of angioectasia $(P=0.0017$ and $P=0.014$, respectively) and erosions/ulcerations $(P=0.0012$ and $P=0.0094$, respectively). Although the detectability of small-bowel lesions by conventional CE $(P=0.020)$ and under FICE setting $2(P=0.0023)$ was reduced by the presence of bile-pigments, that under FICE setting 1 was not affected $(P=0.59)$. Our results also revealed that in poor bowel visibility conditions, CE-FICE yielded a high rate of false-positive findings.
\end{abstract}

Conclusions: CE-FICE may reduce the bile-pigment effect and improve the detectability of small-bowel lesions by CE trainees; the reliability of CE-FICE may be improved by good bowel preparation.

Keywords: Capsule endoscopy (CE), Computed virtual chromoendoscopy system, Flexible spectral imaging color enhancement (FICE), Small bowel

\footnotetext{
*Correspondence: endo1978@yokohama-cu.ac.jp

1 Gastroenterology Division, Yokohama City University School of Medicine, 3-9

Fuku-ura, Kanazawa-ku, Yokohama 236-0004, Japan

Full list of author information is available at the end of the article
} 


\section{Background}

Capsule endoscopy (CE) was introduced in the year 2000 and has since come to be recognized as a useful tool for assessment of the entire small bowel [1-7]. Several studies have reported a higher diagnostic yield of $\mathrm{CE}$ as compared to that of other modalities, such as push enteroscopy [8,9], small-bowel radiography [10] and computed tomography [11]; therefore, $\mathrm{CE}$ is now established as the examination modality of first choice for the evaluation of small bowel lesions. However, the diagnostic yield of CE may be reduced when the visibility of the mucosa is impaired by the presence of air bubbles, food residues and bile pigments. Although the visibility may be improved by bowel preparation, the benefits of bowel preparation for $\mathrm{CE}$ still remain controversial [12-15]. Bile pigments contain bilirubin, look yellow and darken the small bowel, thereby impairing the visibility and lesion recognizability of small bowel lesions, especially by CE trainees. Therefore, it would seem important to devise a method(s) to improve the detectability of small bowel lesions in the presence of bile pigments.

There have been reports of the usefulness of the newly developed computed virtual chromoendoscopy system, namely, flexible spectral imaging color enhancement (FICE), for identifying and diagnosing lesions of the gastrointestinal tract, such as of the esophagus, stomach and large intestine [16-19]. The FICE technology decomposes images by three specific wavelengths (red, green and blue) and then directly reconstructs the images with enhanced surface contrast, thereby improving the visibility of the endoscopic images [19]. The FICE software has recently been incorporated into the new RAPID 6.0 video CE workstation (Given Imaging Ltd, Yoqneam, Israel). With this innovation, the assessor can easily select between conventional images and images reconstructed under three different FICE settings by the click of an icon in the Rapid Reader software for optimal mucosal visualization [20]. Despite the usefulness of CEFICE, it is not widely used in routine practice, because its clinical usefulness is yet to be firmly established.

FICE has been reported to enhance the visibility of small bowel lesions in CE [21], therefore, we predicted that CE-FICE might also allow improved detectability of lesions under conditions of poor bowel visibility, such as in the presence of bile pigments. First, we attempted to confirm whether CE-FICE improved the detectability of small bowel lesions, such as angioectasia and erosions/ ulcerations. Then, we evaluated whether the presence of bile pigments might affect the small bowel lesion detection rate by $\mathrm{CE}$ trainees and whether CE-FICE might allow reduction of the bile pigment effect. In addition, we evaluated whether poor bowel preparation might affect the accuracy of lesion recognition by $\mathrm{CE}$ trainees in CE-FICE. Our results will help to elucidate the clinical usefulness of CE-FICE.

\section{Methods}

\section{Patients}

Of the 98 patients who underwent CE between February 2010 and March 2011 at Yokohama City University School of Medicine, CE expert selected 12 patients who had various lesions of the small bowel and they were enrolled in this study. All of the 12 patients had undergone CE for determination of the cause of obscure gastrointestinal bleeding. The study protocol was approved by the Yokohama City University Hospital Ethics Committee. Written informed consent was obtained from all the subjects prior to their participation in the study. The patients were instructed to swallow the CE capsule (PillCam SB2; Given Imaging Ltd) with a solution of dimethicone after overnight fasting without any other preparation. The patients were allowed to drink clear liquids at 2 hours and eat light meals at 4 hours after swallowing the capsule. Two CE experts (with experience of reporting more than $150 \mathrm{CE}$ videos) separately read and interpreted the complete $\mathrm{CE}$ videos of the 12 patients. To confirm the diagnostic accuracy, intraobserver differences were calculated. If discrepancies were observed, a consensus was reached after the findings were reviewed simultaneously by both the $\mathrm{CE}$ experts.

\section{CE trainees}

Four gastroenterology trainees without $\mathrm{CE}$ experience (A, B, C, and D) were asked to read and interpret the 12 CE videos. All the trainees had performed at least 500 gastroscopies and 50 colonoscopies. They were given the same introduction to the $\mathrm{CE}$ reporting software and given lectures on the typical findings of small bowel lesions (angioectasia and erosions/ulcerations). Prior to this study, they had read one $\mathrm{CE}$ video and were given comprehensive feedback by the $\mathrm{CE}$ experts.

\section{FICE technology for CE}

The principle of the FICE estimation technology is described elsewhere [19]. In brief, this technology takes an ordinary endoscopic image and arithmetically processes the reflected photons for three different wavelengths (red, green and blue) to reconstitute virtual images. The wavelengths for the FICE settings used in the CE evaluation were as follows; setting 1 (red $595 \mathrm{~nm}$, green $540 \mathrm{~nm}$, blue $535 \mathrm{~nm}$ ), setting 2 (red $420 \mathrm{~nm}$, green $520 \mathrm{~nm}$, blue $530 \mathrm{~nm}$ ), setting 3 (red $595 \mathrm{~nm}$, green $570 \mathrm{~nm}$, blue $415 \mathrm{~nm}$ ) (Figure 1). These three different settings made it possible to flexibly select the most suitable wavelengths required for the evaluation of the capsule videos. 

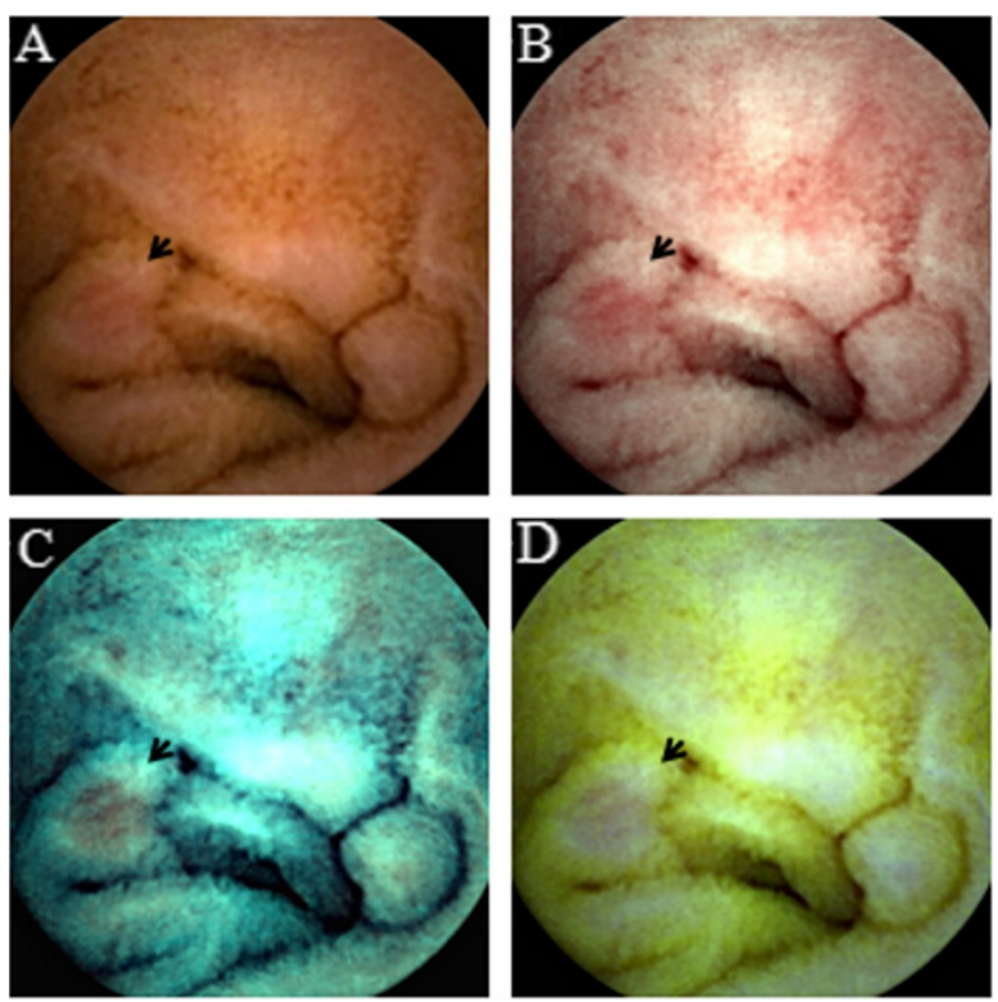

Figure 1 Example of an erosive lesion. The lesion is more clearly visualized by CE-FICE. A: conventional CE image. B-D: CE-FICE images obtained under three different wavelength settings (B: setting 1, C: setting 2. D: setting 3).

\section{Study design}

CE experts marked the first duodenum image and the first cecum image to identify the small bowel area. To avoid recall bias, all the trainees were requested to read each of the CE videos of the small bowel area using only one of the visualization protocols (conventional or one of the three different FICE settings) and recorded perceived abnormalities. Each CE video was set to be viewed under all the four settings (for example, case1; trainee A: conventional, B: FICE setting 1, C: FICE setting 2, D: FICE setting 3), therefore, as a result, each $\mathrm{CE}$ trainees read 4 videos under the conventional $\mathrm{CE}$ setting, 4 under FICE setting 1, 4 under FICE setting 2, and 4 under FICE setting 3. Diagnoses were determined according to samples of the typical findings of small bowel angioectasia and erosions/ulcerations presented by the CE experts. The findings of the CE experts' were defined as the gold standard. We analyzed the lesion detection rate, as the percentage of lesions, relative to the total number, that were correctly detected by the trainees as compared with that by the experts, for each of the FICE settings. To evaluate whether use of the FICE technology might improve the lesion recognition ability of the $\mathrm{CE}$ trainees, the lesion detection rates under each of the FICE settings was compared with that by conventional CE. In addition, $\mathrm{CE}$ trainees tend to miss small-bowel lesions in the bile- pigment-positive condition, therefore we also evaluated whether the presence of bile pigments reduced the detectability of the lesions by the $\mathrm{CE}$ trainees and whether CE-FICE might contribute to reducing the bile pigment effect. The bile pigment condition was determined by the color values around the small bowel lesions according to the results of the receiver operating characteristic (ROC) analysis (Figure 2). Moreover, we evaluated the frequency of the false-positive findings which were incorrectly thumb-nailed for each visibility grade. False-positive findings were defined as reporting of nonpathological conditions, such as food residues and capillary vessels. According to previous reports $[22,23]$, we scored the visibility using a 4-point qualitative evaluation scale (excellent, good, fair and poor) (Figure 3). Each of the $12 \mathrm{CE}$ videos was divided into four segments of equal length, according to the small-bowel transit time, and landmarks were placed to identify the last 10 minutes of every quartile. CE experts evaluated the visibility of each of the segments of 10 minutes' duration using the 4point qualitative index.

\section{Statistical analysis}

Continuous data are shown as mean $\pm \mathrm{SD}$. ROC analysis was performed to determine whether the bile pigment condition was positive or negative. Differences in the 

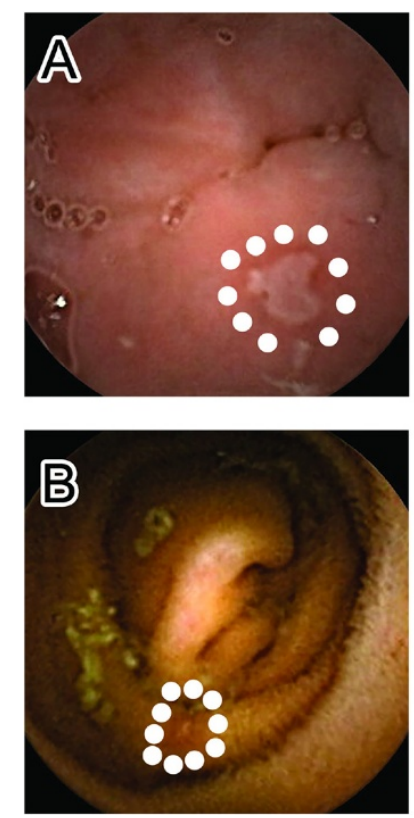

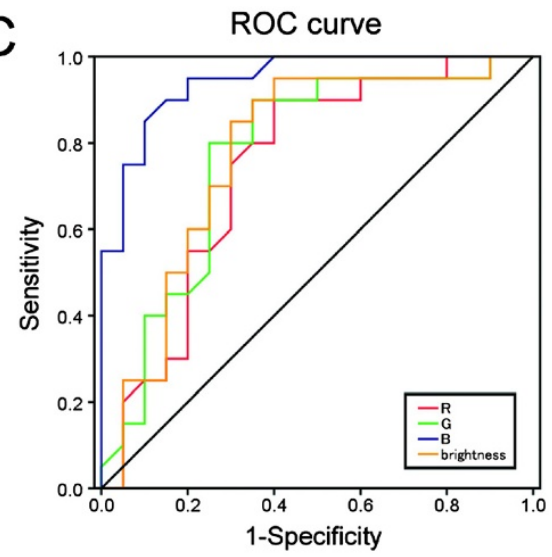

\begin{tabular}{|c|c|c|}
\hline & AUC & $P$ value \\
\hline R & 0.77 & 0.0084 \\
\hline G & 0.77 & 0.0032 \\
\hline B & 0.95 & $<0.0001$ \\
\hline Brightness & 0.78 & 0.0027 \\
\hline
\end{tabular}

Figure 2 Definitions of the bile-pigment-positive and bile-pigment-negative condition. CE experts selected 20 obviously bile-pigmentpositive endoscopic images (A) and 20 bile-pigment-negative endoscopic images (B) that contained small bowel lesions. These images were regarded as golden standard. For each of the images, the RBG (red, blue and green) and brightness (average of ten mucosal points around the small bowel lesions, shown as white dots in this figure) values were calculated. To evaluate the most useful factor for determining whether or not bile pigments are present, a receiver operating characteristic $(\mathrm{ROC})$ analysis was performed $(\mathbf{C})$. The curve was obtained by calculating the sensitivity and specificity of the test at every possible cutoff point, and plotting the sensitivity against 1-specificity. The sensitivity was defined as the proportion of bile-pigment-positive images, determined from each of the color values (RBG and brightness value). The specificity was defined as the proportion of bile-pigment-negative images, also determined from the color values. As shown in Table, the results revealed that the $B$ value was the most useful factor to determine the bile pigment condition (area under the curve (AUC): $0.95, P<0.0001$ ).

lesion detection rates by $\mathrm{CE}$ trainees between conventional $\mathrm{CE}$ and $\mathrm{CE}$ under each of the FICE settings were calculated by the chi-squared test. Differences in the frequency of false-positive findings between conventional $\mathrm{CE}$ and $\mathrm{CE}$ using each of the FICE settings according to the status of bowel visibility were calculated by the Mann-Whitney $U$ test. Intra-observer differences were calculated by kappa statistics.

\section{Results}

\section{Small bowel lesions detected by CE experts}

$\mathrm{CE}$ experts detected 142 lesions of the small bowel, including angioectasia $(\mathrm{n}=60)$ and erosions/ulcerations $(n=82)$. There was a good level of consistency between the two CE experts' findings, with a value of 0.66 .

\section{Lesion detection rates by $\mathrm{CE}$ trainees}

The results are shown in Table 1 . Of a total of 60 angioectasias, CE trainees identified 26 by conventional CE, 40 under FICE setting 1, 38 under FICE setting 2, and 31 under FICE setting 3. Of a total of 82 erosions/ulcerations, $\mathrm{CE}$ trainees identified 38 by conventional $\mathrm{CE}, 62$ under FICE setting 1, 60 under FICE setting 2, and 20 under FICE setting 3. As compared to conventional $\mathrm{CE}, \mathrm{CE}$ under FICE settings 1 and 2 significantly improved the detectability of angioectasia $(P=0.0017$ and $P=0.014$, respectively) and also erosions/ulcerations $(P=0.0012$ and $P=0.0094$, respectively). On the other hand, FICE setting 3 did not allow improved detectability of angioectasia and significantly reduced the detectability of erosions/ulcerations $(P=0.015)$.

\section{Definition of the bile pigment condition}

The color patterns of the endoscopic images were constructed by the wavelengths of three colors (red, green and blue) and the brightness values. Therefore, we hypothesized that these color values and brightness around the small bowel lesions may contribute to determine of the bile pigment condition. First, we selected 20 obviously bile-pigment-positive endoscopic images and 20 bile-pigment-negative endoscopic images containing small bowel lesions (Figure 2). These images were regarded as golden standard. Subsequently, for each of the images, the RBG (red, blue and green) values and brightness (average of ten mucosal points around the small bowel lesion) were calculated. The RBG values and brightness were assessed with a digital scale in the range of $0-255$. To evaluate the factor that might be the most useful for determining the presence of bile pigments, ROC analysis was performed. ROC analysis revealed that 

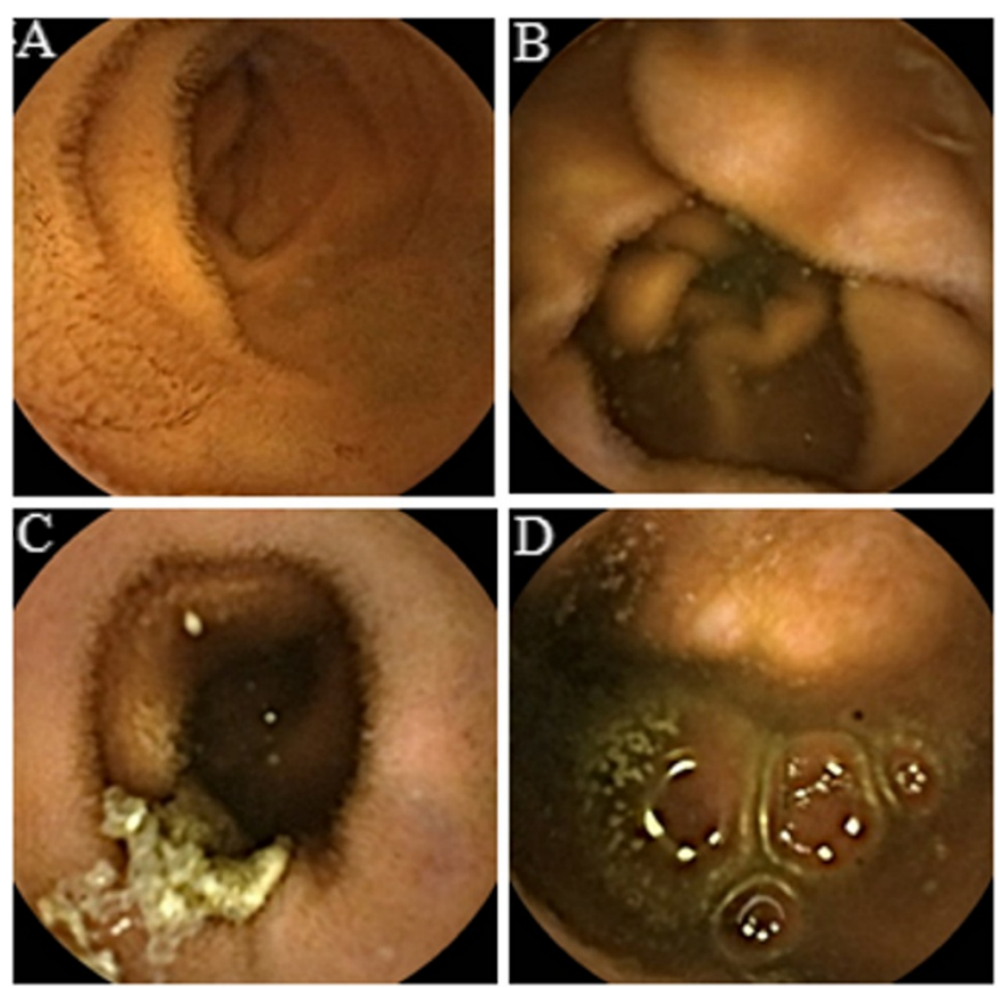

Figure 3 Definition of bowel visibility. Visibility was evaluated using a 4-point qualitative evaluation scale: $\mathbf{A}=$ excellent, visualization of $\geq 90 \%$ of the mucosa, no or minimal fluid, debris and bubbles; $\mathbf{B}=$ good, visualization of $\geq 90 \%$ of the mucosa, mild fluid, debris and bubbles; $\mathbf{C}=$ fair, visualization of $<90 \%$ of the mucosa, moderate fluid, debris and bubbles; $\mathbf{D}=$ poor, visualization of $<80 \%$ of the mucosa, excessive fluid, debris and bubbles.

the $B$ value was the most useful factor to determine the presence/absence of bile pigments (area under the curve (AUC): $0.95, P<0.0001$ ) (Figure 2). Based on this finding, we set the cutoff value at 71.8 (likelihood ratio: 8.5 ); thus, endoscopic images with average $B$ values of lower than 71.8 were defined as bile-pigment-positive images. Use of this cutoff value allowed classification of the endoscopic images, even where it was difficult to determine the bile pigment condition subjectively. As a result, the bile pigment condition of the remaining 102 images was determined by this guideline.

\section{Lesion detection rates by $\mathrm{CE}$ trainees according to the bile pigment condition}

Results are shown in Figure 4. Presence of bile pigments significantly reduced the detectability of small bowel lesions by both conventional $\mathrm{CE}$ and $\mathrm{CE}$ under FICE settings 2 and $3(P=0.02$ and $P=0.0023$, respectively). On the other hand, under FICE setting 1 , the detectability of these lesions was not affected by the presence of bile pigments $(P=0.59)$. Under the bile-pigment-positive condition, the detectability of small bowel lesions was significantly improved by the use of FICE setting 1 $(P=0.043)$, but not that of setting 2 or 3 , as compared with that by the conventional visualization method.

\section{Number of false-positive findings per visibility score}

The results are shown in Table 2. In the presence of poor bowel visibility, the frequency of false-positive findings was significantly increased under FICE settings 2 and 3 , as compared with that in conventional $\mathrm{CE}$ $(P=0.046$ and $P=0.024$, respectively).

Table 1 Evaluation of the lesion detection rate by the CE trainees for each FICE setting

\begin{tabular}{lccccc}
\hline & $\begin{array}{l}\text { Lesions detected } \\
\text { by the CE experts (\%) }\end{array}$ & Conventional & Setting $\mathbf{1}$ & Setting $\mathbf{2}$ & Setting 3 \\
\hline Angioectasia & $60(100)$ & $26(43.3)$ & $40(66.7)^{*}$ & $38(63.3)^{*}$ & $31(51.7)$ \\
Erosion/ulceration & $82(100)$ & $38(46.3)$ & $62(75.6)^{\dagger}$ & $60(73.2)^{\dagger}$ & $20(24.4)^{*}$ \\
\hline
\end{tabular}

${ }^{\circ}$ Comparison of conventional CE with FICE settings 1 and 2 by the chi-squared test $(P=0.0017$ and $P=0.014$, respectively).

${ }^{+}$Comparison of conventional CE with FICE settings 1 and 2 by the chi-squared test $(P=0.0012$ and $P=0.0094$, respectively).

tComparison of conventional CE with FICE setting 3 by the chi-squared test $(P=0.015)$. 


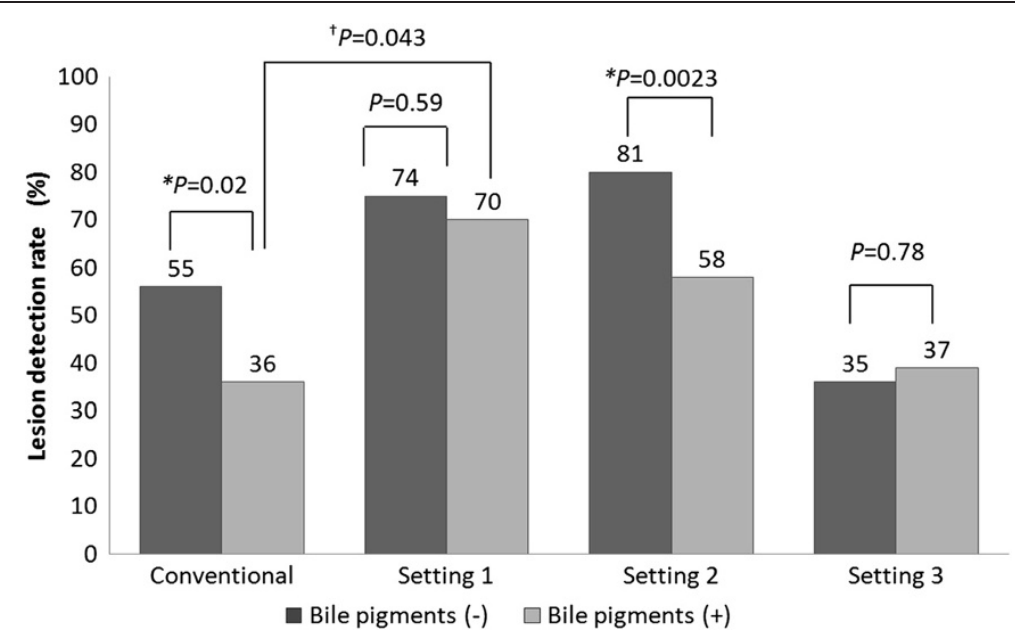

Figure 4 Evaluation of the lesion detection rate according to the bile pigment condition. The lesion detection rate under the conventional CE setting and FICE setting 2 were significantly reduced in the presence of bile pigments in the small bowel $(*)$. On the other hand, the lesion detection rate under FICE setting 1 was not affected by the presence of bile pigments. In the bile-pigment-positive condition, the lesion detection rate under FICE setting 1 was significantly higher than that by conventional CE $\left(^{\dagger}\right)$.

\section{Discussion}

Although the lesion recognition ability of the CE trainees enrolled in our study was low, consistent with previous reports [24-27], use of FICE setting 1 or 2 improved the detectability of angioectasia and erosions/ ulcerations by the CE trainees. Imagawa et al. reported from a study of CE experts, that CE-FICE improved the detectability of angioectasia, but not that of erosions/ ulcerations [28]. Contrary to their results, the detectability of erosions/ulcerations was also improved by CEFICE in our study. Although CE trainees are often likely to miss small erosions/ulcerations [24,25], FICE enhances the visibility of these lesions, making them easy to recognize by even relatively untrained eyes.

Table 2 Evaluation of the number of false-positive findings for each visibility score

\begin{tabular}{lccccc}
\hline & $\begin{array}{l}\text { No. of } \\
\text { Segments }\end{array}$ & \multicolumn{2}{l}{ No. of false positive findings/segment } \\
\cline { 4 - 7 } & Conventional Setting $\mathbf{1}$ Setting 2 Setting 3 \\
\hline Visibility score & & & & & \\
excellent & 7 & $0.1 \pm 0.4$ & $0.1 \pm 0.4$ & $0.3 \pm 0.5$ & $0.4 \pm 0.5$ \\
good & 13 & $0.3 \pm 0.1$ & $0.2 \pm 0.6$ & $0.2 \pm 0.4$ & $0.5 \pm 0.7$ \\
fair & 20 & $0.6 \pm 0.8$ & $0.9 \pm 1.0$ & $1.2 \pm 1.1$ & $1.7 \pm 1.4^{*}$ \\
poor & 8 & $0.9 \pm 0.6$ & $1.6 \pm 0.9$ & $2.9 \pm 1.9^{\dagger}$ & $2.6 \pm 1.8^{\dagger}$ \\
\hline
\end{tabular}

NOTE: Each of the $12 \mathrm{CE}$ videos was divided into four segments of equal length, according to the small bowel transit time, and landmarks were placed to identify the last 10 minutes of every quartile. The $C E$ experts evaluated the visibility of the each segment of 10 minutes' duration using a 4-point qualitative index (excellent, good, fair and poor).

Values are mean \pm SD.

"Comparison of conventional CE with FICE setting 3 by the Mann-Whitney $U$ test $(P=0.018)$.

${ }^{+}$Comparison of conventional CE with FICE settings 2 and 3 by the Mann-Whitney $U$ test $(P=0.046$ and $P=0.024)$.
Therefore, the detectability of erosions/ulcerations might also be improved by the use of FICE in CE trainees. Angioectasia and erosions/ulcerations represent the major sources of bleeding from the small bowel $[29,30]$, therefore, CE-FICE could be useful for diagnosis of the cause of obscure GI bleeding.

To investigate how CE-FICE might improve the detectability of small bowel lesions, the influence of the presence of bile pigments on the detectability of the small bowel lesions was first evaluated. In our study, the results of the ROC curve analysis revealed that the bile pigment condition was best determined by the $B$ values around the small bowel lesions. As the $\mathrm{B}$ values became lower, the images began to look more yellow. Therefore, we concluded that determination of the $B$ value to evaluate the bile pigment condition was reasonable. Our results indicated that while the $\mathrm{CE}$ trainees were likely to miss small bowel lesions in the presence of bile pigments, FICE setting 1 appeared to improve the lesion recognizability under this condition. Therefore, use of FICE setting 1 may be clinically meaningful in the bilepigment-positive condition. Similar to the case for the $\mathrm{CE}$ trainees, the presence of bile pigments may affect the detectability of small bowel lesions even by doctors with experience in CE. To verify this contention, however, additional studies are needed.

Our results suggested that CE-FICE requires extra caution under the condition of poor bowel preparation. CE trainees tend to confuse food residues with erosions/ ulcerations, and capillary vessels with angioectasia, especially under FICE settings 2 and 3. This indicates that good bowel preparation is preferable for assessing $\mathrm{CE}$ videos using FICE. 


\section{Limitations}

Although our results indicated the potential clinical usefulness of CE-FICE, there were some limitations of this study. First, we compared the lesion detection rates by the $\mathrm{CE}$ trainees, under conventional and three different FICE settings with the findings in the same CE exam previously read by $\mathrm{CE}$ experts. The trainees were requested to read each of the $\mathrm{CE}$ videos under only any one of the visualization protocols. Although this method might help to avoid recall bias, it dose not provide accurate measurement of the diagnostic accuracy or diagnostic improvement. Second, there is no gold standard at present to confirm whether the area analyzed by RBG image analysis indeed has bile pigments. It is necessarily based on subjective evaluation by the examiner. Third, some lesions reported as false-positives could correspond to new lesions only seen with FICE settings and not necessarily actually false positive. The difficulties in resolving these biases are potential flaws of this study.

\section{Conclusion}

Based on a study of $\mathrm{CE}$ trainees, we demonstrated that the detectability of small bowel angioectasia and erosions/ulcerations could be improved by CE-FICE (FICE settings 1 and 2). Moreover, we showed that FICE setting 1 enabled reduction of the bile pigments effect and improved the detectability of small bowel lesions. In addition, our results also indicated that good bowel preparation is important for assessment by CE-FICE.

\section{Abbreviations \\ CE: Capsule endoscopy; FICE: Flexible spectral imaging color enhancement.}

\section{Competing interests}

The authors have no competing interests to declare.

\section{Authors' contributions}

ES, HE and HT designed the study. ES wrote the article. $\mathrm{KH}, \mathrm{EY}, \mathrm{HO}$ and $\mathrm{TH}$ participated in the critical revision of the manuscript. TM, WT, LT and TU participated in the study as CE trainees and interpreted the CE videos. SK performed the statistical analysis. $\mathrm{HE}, \mathrm{YH}$ and $\mathrm{AN}$ were responsible for the design of the study. All authors read and approved the submission of the final manuscript.

\section{Authors' information}

ES and $\mathrm{HE}$ are physicians specializing in small bowel diseases, and belong to the Gastroenterology division, Yokohama City University School of Medicine, 3-9 Fuku-ura, Kanazawa-ku, Yokohama, 236-0004 Japan.

\section{Acknowledgements}

The authors thank Ryo Takahashi for his technical support with the optical endoscopic technology.

\section{Author details}

${ }^{1}$ Gastroenterology Division, Yokohama City University School of Medicine, 3-9 Fuku-ura, Kanazawa-ku, Yokohama 236-0004, Japan. ${ }^{2}$ Gastroenterology Division, Chigasaki City Hospital, Chigasaki, Japan.

Received: 16 March 2012 Accepted: 19 June 2012 Published: 2 July 2012
References

1. Iddan G, Merson G, Glukhovsky A, Swain P: Wireless capsule endoscopy. Nature 2000, 405:17

2. Meron GD: The development of the swallowable video capsule (M2A) Gastrointest Endosc 2000, 52:817-9.

3. Lewis BS, Swain P: Capsule endoscopy in the evaluation of patients with suspected small intestinal bleeding: results of a pilot study. Gastrointest Endosc 2002, 56:349-53.

4. Pennazio M, Santucci R, Rondonotti E, Abbiati C, Beccari G, Rossini FP, De Franchis $R$ : Outcome of patients with obscure gastrointestinal bleeding after capsule endoscopy; report of 100 consecutive cases. Gastroenterology 2004, 126:643-53.

5. Apostolopoulos P, Liatosos C, Gralnek IM, Giannakoulopoulou E, Alexandrakis G, Kalantzis C, Gabriel P, Kalantzis N: The role of wireless capsule endoscopy in investigating unexplained iron deficiency anemia after negative endoscopic evaluation of the upper and lower gastrointestinal tract. Endoscopy 2006, 38:1127-32.

6. Endo H, Hosono K, Inamori M, Kato S, Nozaki Y, Yoneda K, et al: Incidence of small bowel injury induced by low-dose aspirin: a crossover study using capsule endoscopy in healthy volunteers. Digestion 2009, 79:44-51.

7. Endo H, Hosono K, Inamori M, Nozaki Y, Yoneda K, Fujita K, Takahashi H, Yoneda M, Abe Y, Kirikoshi H, Kobayashi N, Kubota K, Saito S, Ohya T, Hisatomi K, Teratani T, Matsuhashi N, Nakajima A: Characteristics of small bowel injury in symptomatic chronic low-dose aspirin users: the experience of two medical centers in capsule endoscopy. J Gastroenterol 2009, 44:544-9.

8. Mylonaki M, FritscheRavens A, Swain P: Wireless capsule endoscopy: a comparison with push enteroscopy with gastroscopy and colonoscopy negative gastrointestinal bleeding. Gut 2003, 52:1122-6.

9. Saurin JC, Delvaux M, Gaudin JL, Fassler I, Villareio J, Vahedi K, Bitoun A, Canard JM, Souquet JC, Ponchon T, Florent C, Gay G: Daiagnostic values of endoscopic capsule in patients obscure bleeding: blinded comparison with video push-enteroscopy. Endoscopy 2003, 35:576-84.

10. Constamagna G, Shah SK, Riccioni ME, Foschia F, Mutignani M, Perri V, Vecchioli A, Brizi MG, Picciocchi A, Marano P: A prospective trial comparing small bowel radiographs and video capsule endoscopy for suspected small bowel disease. Gastroenterology 2002, 123:999-1005.

11. Saperas E, Dot J, Videla S, Alvarez-Castells A, Perez-Lafuente M, Armengol JR, Malagelada JR: Capsule endoscopy versus computed tomography or standard angiography for the diagnosis of obscure gastrointestinal bleeding. Am J Gastroenterol 2007, 102:731-7.

12. Viazis N, Sgouros S, Papaxoinis K, Vlachogiannakos J, Bergele C, Sklavus P, Panari A, Avgerinos A: Bowel preparation increases the diagnostic yield of capsule endoscopy: A prospective, randomized, controlled study. Gastrointest Endosc 2004, 60:534-8.

13. Endo $H$, Kondo $Y$, Inamori $M$, Ohya TR, Yanagawa $T$, Asayama M, Hisatoshi $K$, Teratani T, Yoneda M, Nakajima A, Matsuhashi N: Ingesting $500 \mathrm{ml}$ polyethylene glycol solution during capsule endoscopy improves the image quality and completion rate to the cecum. Dig Dis Sci 2008, 53:3201-5

14. Ben-Soussan E, Savoye G, Antonietti M, Ramirez S, Ducrotte P, Levebours E: Is a 2-liter PEG preparation useful before capsule endoscopy? J Clin Gastroenterol 2005, 39:381-4.

15. Franke A, Hummel F, Knebel P, Antoni C, Bocker U, Singer MV, Lohr M: Prospective evaluation of small bowel preparation with bisacodyl and sodium phosphate for capsule endoscopy. World J Gastroenterol 2008, 14:2061-4

16. Pohl J, May A, Rabenstein T, Pech O, Nguyen-Tat M, Fissler-Eckhoff A, Ell C: Comparison of computed virtual chromoendoscopy and conventional chromoendoscopy with acetic acid for detection of neoplasia in Barrett's esophagus. Endoscopy 2007, 39:594-8.

17. Pohl J, Nguyen-tat M, Pech O, May A, Rabenstein T, Ell C: Computed virtual chromoendoscopy for classification of small colorectal lesions; a prospective comparative study. Am J Gastroenterol 2008, 103:562-9.

18. Mouri R, Yoshida S, Tanaka S, Oka S, Yoshihara M, Chayama K: Evaluation and validation of computed virtual chromoendoscopy in early gastric cancer. Gastrointest Endosc 2009, 69:1052-8.

19. Pohl J, May A, Rabenstein T, Pech O, Ell C: Computed virtual chromoendoscopy: a new tool for enhancing tissue surface structures. Endoscopy 2007, 39:80-3. 
20. Pohl J, Ashmoneit I, Schuhmann S, Ell C: Computed image modification for enhancement of small-bowel surface structures at video capsule endoscopy. Endoscopy 2010, 42:490-2.

21. Imagawa H, Oka S, Tanaka S, Noda I, Higashiyama M, Sanomura Y, Shishido T, Yoshida S, Chayama K: Improved visibility of lesions of the small intestine via capsule endoscopy with computed virtual chromoendoscopy. Gastrointest Endsc 2011, 73:299-306.

22. Brotz C, Nandi N, Conn M, Daskalakis C, DiMarino M, Infantolino A, Katz LC, Schroeder T, Kastenberg D: A validation study of 3 grading systems to evaluate small-bowel cleaning for wireless capsule endoscopy: a quantitative index, a qualitative evaluation, and an overall adequacy assessment. Gastrointest Endosc 2009, 69:262-70.

23. VanWeyenberg SJ, De Leest HT, Mulder CJ: Description of a novel grading system to assess the quality of bowel preparation in video capsule endoscopy. Endoscopy 2011, 43:406-11.

24. Postgate A, Haycock A, Thomas-Gibson S, Fitzpatrick A, Bassett P, Preston S, Saunders BP, Fraser C: Computer-aided learning in capsule endoscopy leads to improvement in lesion recognition abilty. Gastrointest Endosc 2009, 70:310-6.

25. Sidhu R, Sakellariou P, McAlindon ME, Leeds JS, Shafiq K, Hoeroldt BS, Hopper AD, Karmo M, Salmon C, Elphick D, Ali A, Saunders DS: Is formal training necessary for capsule endoscopy? The largest gastroenterology trainee study with controls. Dig Liver Dis 2008, 40:298-302.

26. Levinthal GN, Burke CA, Statisi JM: The accuracy of an endoscopy nurse in interpreting capsule endoscopy. Am J Gastroenterol 2003, 98:2669-71.

27. Bossa F, Cocomazzi G, Valvano MR, Andriulli A, Annesa V: Detection of abnormal lesions recorded by capsule endoscopy. A prospective study comparing endoscopist's and nurse's accuracy. Dig Liver Dis 2006, 38:599-602.

28. Imagawa H, Oka S, Tanaka S, Noda I, Higashiyama M, Sanomura Y, Shishido T, Yoshida S, Chayama K: Improved detectability of small-bowel lesions via capsule endoscopy with computed virtual chromoendoscopy: A pilot study. Scand I Gastroenterol 2011, 46:1133-7.

29. Heinz GD, Hadithi M, Groenen MJ, Kuipers EJ, Jacobs MA, Mulder CJ: Double-balloon enteroscopy: indications, diagnostic yield, and complications in a series of 275 patients with suspected small-bowel disease. Endoscopy 2006, 38:42-8.

30. Ohmiya N, Yano Y, Yamamoto H, Arakawa D, Nakamura M, Honda W, Itoh A, Hirooka Y, Niwa Y, Maeda O, Ando T, Yao T, Matsui T, lida M, Tanaka S, Chiba T, Sakamoto C, Sugano K, Goto H: Diagnosis and treatment of obscure Gl bleeding at double balloon endoscopy. Gastrointest Endosc 2007, 66:72-7.

doi:10.1186/1471-230X-12-83

Cite this article as: Sakai et al:: Capsule endoscopy with flexible spectral imaging color enhancement reduces the bile pigment effect and improves the detectability of small bowel lesions. BMC Gastroenterology 2012 12:83.

\section{Submit your next manuscript to BioMed Central and take full advantage of:}

- Convenient online submission

- Thorough peer review

- No space constraints or color figure charges

- Immediate publication on acceptance

- Inclusion in PubMed, CAS, Scopus and Google Scholar

- Research which is freely available for redistribution 